\title{
Editorial for the special issue mODa12: Advances in Model-Oriented Design and Analysis
}

\author{
Radoslav Harman ${ }^{1} \cdot$ Werner G. Müller ${ }^{2}$. David Woods ${ }^{3}$ \\ Published online: 19 March 2019 \\ ๑) Springer-Verlag GmbH Germany, part of Springer Nature 2019
}

The international workshops on model-oriented data analysis and optimum design (mODa) provide a high-level forum for researchers and practitioners to present and discuss recent advances, new techniques and applications in the field of optimum experimental design. An important characteristic of the mODa series is that participation is solely by invitation through the board, there are no parallel sessions, and all presentations are of the same length. A primary aim of the workshops is to offer young researchers an opportunity to establish personal contacts with leading specialists in the field.

The mODa series started in 1987, when the first workshop was held at the Wartburg Castle, Eisenach, in the former German Democratic Republic, initiated by V.V. Fedorov and H. Läuter. Since then, it has been organized every 3 years (with one exception) at various locations throughout Europe, and has become the leading conference series on optimum design of experiments and related areas. The 12th meeting will be held at Smolenice Castle, Slovak Republic from 23rd to 28th of June 2019, organized by Radoslav Harman and Viktor Witkovský.

As mODa has now come of age, the board in the last revision of its charter expressed the hope that traditions of collaboration and friendship going back for more than 20 years can be passed onto new generations of statisticians and act as a springboard for future joint research activities. To reflect the maturity of the workshops, the mODa board has nominated three lifetime invitees to honour their achievements for the mODa idea, namely Anthony C. Atkinson, Andrej Pázman and Ben Torsney.

The proceedings of these workshops have traditionally been published and made available at the event, mostly in a Lecture Notes Series of Physica by Springer-Verlag (see the chronological list in the References section). At the last workshop the mODa board decided to take up an offer by Statistical Papers (also published by Springer-

\footnotetext{
$凶$ Radoslav Harman harman@fmph.uniba.sk

1 Comenius University in Bratislava, Bratislava, Slovakia

2 Johannes Kepler University Linz, Linz, Austria

3 University of Southampton, Southampton, UK
} 
Verlag) to instead publish a special issue of the journal. The following papers reflect the work presented and discussed at mODa 12.

(1) Mielke and Dragalin study adaptive designs for drug combination with a binary efficacy endpoint. They consider the situation when the timing of the endpoint assessment period on the subject level is long relative to the inter-arrival time of subjects. The difficulties that arise for the adaptive design problem in this case are solved by using an exponential time-to-event models in the interim analysis to reassess the study design. It is shown that the use of this longitudinal model can improve the information for interim decision making.

(2) Tarima and Flournoy consider an experiment in which the primary objective is to determine the significance of a treatment effect at a predetermined type I error and statistical power, and the secondary objective is to estimate the treatment effect. They study the asymptotic behaviour of the full-data maximum likelihood estimator following sample size re-estimation. Their main result states that the asymptotic distributions of standardized statistics are random mixtures of distributions, which are non-normal except under certain model choices for sample size re-estimation.

(3) Wang, Rosenberger and Uschner address the problem of testing the significance of treatment effects in randomized clinical trials. Through simulations, the authors justify distinguishing between randomization-based inference and population-based parametric and nonparametric inference. The dynamic of selecting an appropriate randomization procedure with respect to the research aim and the clinical circumstance is shown by studying the power of randomization tests. It is proposed that the choice of randomization procedures and the analysis of randomized clinical trials can be facilitated by the application of randomization-based inference.

(4) Bailey and Cameron consider basket trials, where several different drugs are tested on several different diseases in a single protocol which involves many medical centres. Specifically, the paper deals with designs for cancer trials which allow each medical centre to treat only a limited number of cancer types with only a limited number of drugs. Desirable properties of these designs are discussed, and several different methods of construction are given.

(5) The paper of Filipiak, Khodsiani and Markiewicz focuses on optimal properties of circular balanced block designs under the model with circular autoregression of the first order. The authors provide results on universal optimality of balanced block designs with equal block sizes. Moreover, they show E-optimality of complete balanced block designs with the number of blocks equal to the number of treatments or the number of treatments equal to the number of blocks minus two.

(6) Malevich and Müller find optimum designs for inspection times of continuous lifetime variables. The authors consider optimal equidistant and optimal nonequidistant inspection times for interval censoring of exponential distributions, for which a recursive formula is provided. Moreover, the efficiency of optimal equidistant inspection times with respect to optimal non-equidistant inspection 
times is studied and since the optimal inspection times are only locally optimal, some results concerning maximin efficient designs are given.

(7) Next, Prus studies prediction of individual parameters in random-coefficient regression. In this situation, the optimum experimental designs may depend on the unknown covariance structure of the random effects. For selected models, the author provides optimum designs with respect to the minimax integrated mean square error criterion.

(8) Fontana provides formulae to compute the generalized word-length pattern of any subfraction obtained by the deletion of some of the points in an orthogonal array. They provide a simple method to find the best sub-fractions with respect to the generalized minimum aberration criterion, in the case when a single point is deleted. The effect of deleting up to three points is then studied on examples.

(9) The paper by Freise and Schwabe presents analytical results on the D-optimum designs for factorial models with first-order interactions when the number of active factors is symmetrically bounded from below and from above. The approach used for characterizing optimum designs is based on invariance and equivariance properties with respect to natural symmetries in both the model and the design region. The results on the support points also carry over to other criteria based on the eigenvalues of the information matrix.

(10) Radloff and Schwabe develop locally D-optimum designs on a k-dimensional ball for a wide class of non-linear multiple regression problems which include binary response models with logit or probit link. Rotation-invariant approximate designs are obtained which consist of two parallel orbits on the surface of the ball.

(11) The paper by Pázman provides a new approximation of the probability density function of the ordinary least squares estimator in nonlinear regression; the approximation is close to the true density for nonlinear models with small intrinsic curvature. Possible applications in experiments involving heat transfer are indicated.

(12) In their previous work, Pronzato, Wynn and Zhigljavsky defined the k-th order simplicial distance between probability distributions which can be embedded in the wider theory of divergences and distances based on concave functionals. Their paper explores the question of which distance, or which type of concave experimental design criterion, is best able to distinguish between two normal distributions. Among other results, the authors show that Kiefer's family of design criteria may conveniently be used to define distances between normal distributions, in particular for measures concentrated in small dimension subspaces.

(13) The paper by Sagnol and Pauwels explains how the problem of computing Bayes A-optimum designs can be reformulated as an unconstrained convex problem closely related to the well-studied problem of group lasso regression. This reformulation opens a way for using algorithms developed by the optimization community, and the paper demonstrates such a use for several of these algorithms. An appealing property of these methods is that they come with rigorous convergence guaranties, and yield sparse iterates very quickly, corresponding to designs with only a few support points. 
(14) To meet the challenge of massive data, Yao and Wang developed an optimal subsampling method for softmax regression which is commonly used to model data with multiple categorical responses. Optimal subsampling probabilities under A- and L-optimality criteria are derived, and the asymptotic normality of the general subsampling estimator is established.

\section{References}

Fedorov V, Läuter H (eds) (1987) Model-oriented data analysis: proceedings of an IIASA workshop on data analysis held at Eisenach, GDR, in March 1987, Lecture notes in economics and mathematical systems (Book 297). Springer, Berlin

Fedorov VV, Müller WG (1992) Model oriented data-analysis: a survey of recent methods : proceedings of the 2nd IIASA-workshop in St. Kyrik, Bulgaria, May 28-June 1, 1990. Springer, Heidelberg

Müller WG, Wynn HP, Zhigljavsky AA (1993) Model-oriented data analysis: proceedings of the 3rd international workshop in Petrodvorets, Russia, May 25-30, 1992. Physica, Heidelberg

Kitsos CP, Müller WG (eds) (1995) MODA4: advances in model-oriented data analysis, contributions to statistics. Physica, Heidelberg. https://doi.org/10.1007/978-3-662-12516-8

Atkinson AC, Pronzato L, Wynn HP (eds) (1998) MODA 5: advances in model-oriented data analysis and experimental design, contributions to statistics. Physica, Heidelberg. https://doi.org/10.1007/978-3642-58988-1

Atkinson AC, Hackl P, Müller WG (eds) (2001) mODa 6: advances in model-oriented design and analysis, contributions to statistics. Physica, Heidelberg. https://doi.org/10.1007/978-3-642-57576-1

Di Bucchianico A, Läuter H, Wynn HP (eds) (2004) mODa 7: advances in model-oriented design and analysis, contributions to statistics. Physica, Heidelberg. https://doi.org/10.1007/978-3-7908-2693-7

López-Fidalgo J, Rodríguez-Díaz JM, Torsney B (eds) (2007) mODa 8: advances in model-oriented design and analysis, contributions to statistics. Physica, Heidelberg. https://doi.org/10.1007/978-37908-1952-6

Giovagnoli A, Atkinson AC, Torsney B, May C (eds) (2010) mODa 9: advances in model-oriented design and analysis, contributions to statistics. Physica, Heidelberg. https://doi.org/10.1007/978-3-7908-2410-0

Ucinski D, Atkinson AC, Patan M (eds) (2013) mODa 10: advances in model-oriented design and analysis, contributions to statistics. Springer, Heidelberg. https://doi.org/10.1007/978-3-319-00218-7

Kunert J, Müller CH, Atkinson AC (eds) (2016) mODa 11: advances in model-oriented design and analysis, contributions to statistics. Springer, Cham. https://doi.org/10.1007/978-3-319-31266-8

Publisher's Note Springer Nature remains neutral with regard to jurisdictional claims in published maps and institutional affiliations. 